Reprod. Nutr. Dévelop., 26 (1 B), 351-352.

\title{
Influence du mode d'élevage et du poids d'abattage sur les caractéristiques des lipides musculaires de l'agneau de boucherie
}

\author{
B. AUROUSSEAU, P. VIGNERON $\left({ }^{*}\right)$
}

\begin{abstract}
Laboratoire d'Etude du Métabolisme énergétique, I.N.R.A., Theix, 63122 Ceyrat France. Laboratoire de Physiologie animale, I.N.R.A., 34060 Montpellier Cédex.
\end{abstract}

Summary. Mean muscle TG content was increased $(P<0.025)$ from 17 up to $20 \mathrm{mg} / \mathrm{g}$ in lambs slaughtered at 25 or $32.5 \mathrm{~kg}$ respectively and was similar in lambs grazed on " garrigue " or fed indoor hay and concentrate. Mean muscle PL content $(3.6 \mathrm{mg} / \mathrm{g})$ was altered $(P<0.025)$ by both factors. AGP $(n-6)$ and AGP $(n-3)$ proportions in muscle PL amounted respectively to 22.4 and $12.5 \%$ in grazed lambs or to 27.1 and $6.7 \%$ in indoor fed lambs.

Les lipides musculaires jouent un rôle à la fois sur les qualités diététiques et les qualités organoleptiques des viandes (Cf. Aurousseau, 1979). Nous examinerons ces aspects dans une communication ultérieure, et dans un premier temps, nous rapportons ici quelques résultats préliminaires concernant l'influence du mode d'élevage des agneaux sur les quantités de lipides dans le tissu musculaire et sur leur composition.

Matériel et méthodes. Vingt huit agneaux Mérinos d'Arles nés entre le 15 et le 28 février ont été élevés sous la mère, soit sur des parcours de garrigue, soit en bergerie, ayant dans ce cas accès à un foin de luzerne et à un aliment concentré à base de céréales. La moitié des animaux ont été abattus au poids de $25 \mathrm{~kg}$, l'autre moitié au poids de $32,5 \mathrm{~kg}$. La concentration et la composition en acides gras des triglycérides (TG) et des phospholipides (PL) ont été déterminées, selon un protocole décrit antérieurement (Bauchart et Aurousseau, 1981), sur 3 muscles : le Iongissumus dorsi (LD), le supra-spinatus (SS) et le tensor fasciae latae (TFL).

Résultats et discussion. L'analyse de variance montre que la concentration en TG du muscle n'a pas été influencée par le mode d'élevage, alors que chez les mêmes animaux les tissus adipeux ont été 1,6 fois plus importants chez les agneaux élevés en bergerie (Vigneron, observation non publiées). Au poids de $25 \mathrm{~kg}$, les teneurs en TG des muscles LD $(P<0,05)$ et $S S(P<0,06)$ ont été plus élevées chez les agneaux élevés en bergerie. Par ailleurs, les teneurs observées à $25 \mathrm{~kg}(17 \mathrm{mg} / \mathrm{g})$ ont été inférieures $(\mathrm{P}<0,025)$ à celles observées à $32,5 \mathrm{~kg}$ $(20 \mathrm{mg} / \mathrm{g})$, tandis que les 3 muscles considérés ont présenté des teneurs en TG différentes ( $P<0,005), 11-17$ ou $22 \mathrm{mg} / \mathrm{g}$ pour le $L D$, le $S S$ ou le TFL, respectivement (tabl. 1).

A l'inverse, la concentration en $P L$ du muscle a été inférieure $(P<0,005)$ chez les agneaux élevés en bergerie $(3,3$ contre $3,9 \mathrm{mg} / \mathrm{g})$; elle a été plus élevée $(P<0,01)$ à $25 \mathrm{~kg}(3,8 \mathrm{mg} / \mathrm{g})$ qu'à $32,5 \mathrm{~kg}(3,3 \mathrm{mg} / \mathrm{g})$; elle a également pré- 
senté des variations $(P<0,005)$ d'un muscle à l'autre : $3,1-4,2$ ou $3,4 \mathrm{mg} / \mathrm{g}$ pour le Ld, le SS ou le TFL respectivement (tabl. 1).

TABL. 1. - Effet du mode d'alimentation et du poids de l'Agneau Mérinos d'Arles sur les teneurs du muscle (1) en triglycérides (TG) et en phospholipides (PL) et sur les proportions d'acides gras polyinsaturés (AGP) dans les PL.

\begin{tabular}{lrrrr}
\hline Mode d'élevage & \multicolumn{2}{c}{ Garrigue } & \multicolumn{2}{c}{ Bergerie } \\
\hline Poids des agneaux (kg) & 24,9 & 32,2 & 24,7 & 33,3 \\
Age des agneaux (jours) & 86,6 & 106,6 & 81,6 & 109,3 \\
Gain moyen quotidien (g/j) & 247,0 & 264,8 & 259,9 & 276,9 \\
Teneur en TG (mg/g) & 16,2 & 19,3 & 17,8 & 21,3 \\
Teneur en PL (mg/g) & 4,3 & 3,6 & 3,5 & 3,2 \\
AGP (n-6) des PL (2) & 22,3 & 22,4 & 27,1 & 27,1 \\
AGP (n-3) des PL (2) & 12,8 & 12,1 & 6,9 & 6,5 \\
\hline
\end{tabular}

(1) Moyennes des observations effectuées sur 3 muscles : longissimus dorsi, supra-spinatus, tensor fasciae latae. (2) \% des AG totaux.

Dans les TG, seules les proportions d'acides gras polyinsaturés (AGP) ont été modifiés $(P<0,01)$ par le mode d'élevage des agneaux : chez les agneaux élevés en bergerie, les proportions d'AGP (n-6) ont été supérieures $(3,0$ contre $2,5 \%)$ et les proportions d' AGP $(n-3)$ inférieures $(0,9$ contre $9,6 \%)$. Par ailleurs, les proportions d'AG monoinsaturés ont été plus faibles $(P<0,05)$ chez les agneaux de $25 \mathrm{~kg}(43,5 \%)$ que chez ceux de $32,5 \mathrm{~kg}(47,3 \%)$. Enfin, la composition en acides gras des TG s'est montrée indépendante du muscle considéré.

De même, dans les $P L$, les proportions d'AGP $(n-6)$ ont été supérieures et les proportions d'AGP $(n-3)$ inférieures $(P<0,05 ;$ tabl. 1$)$ dans le cas des agneaux élevés en bergerie $(27,1$ ou $6,7 \%$ respectivement contre 22,4 ou $12,5 \%)$. Par ailleurs, la composition des PL est restée stable entre 25 et $32,5 \mathrm{~kg}$ tandis que les muscles LD et TFL se sont révélés plus riches en $A G$ aliphatiques saturés que le muscle SS $(28,1$ contre $24,9 \%)$.

En conclusion, l'alimentation plus libérale et les dépenses de thermogénèse et de locomotion plus faibles chez les agneaux élevés en bergerie, traduites par ailleurs par des gains de poids supérieurs (tabl. 1) pourraient expliquer les concentrations en TG musculaires sensiblement plus élevées observées au poids de $25 \mathrm{~kg}$ chez ces agneaux. On peut ainsi noter que le développement des tissus adipeux à ce stade est beaucoup plus important chez les mêmes agneaux, tandis qu'entre 25 et $32,5 \mathrm{~kg}$, l'écart entre les 2 groupes a diminué (Vigneron, observations non publiées) et que l'accumulation de TG dans le muscle se comporte de la même façon, mais la différence entre les 2 groupes n'est plus dans ce cas significative à $32,5 \mathrm{~kg}$. En revanche, la composition en acides gras des PL s'est révélée étroite tement liée au mode d'élevage et à la nature des aliments ingérés, puisque chez les agneaux élevés en bergerie, aux 2 poids, les proportions d'AGP (n-6) sont supérieures et celles d'AGP (n-3) plus faibles que chez ceux élevés sur parcours de garrigues.

Aurousseau B., 1979. Influence de la nature des lipides tissulaires sur la qualité des carcasses et des viandes des ruminants. Bull. tech. CRZV Theix, I.N.R.A., 38, $27-34$.

Bauchart D., Arousseau B., 1981. Postprandial lipids in blood plasma of preruminant calves. J. Dairy Sci., 64, 2033-2042. 